\title{
DEVELOPMENT OF TEST RIG SYSTEM FOR CALIBRATION OF TEMPERATURE SENSING FABRIC
}

\author{
Muhammad Dawood Husain ${ }^{1,2, *}$, Ozgur Atalay, ${ }^{1,3}$, Asli Atalay ${ }^{1,4}$ and Richard Kennon ${ }^{1}$
}

${ }^{1}$ School of Materials, University of Manchester, Manchester, M60 1QD, UK.

${ }^{2}$ Textile Engineering Department, NED University of Engineering \& Technology, University Road, Karachi, Pakistan;

3iTU Textile Technologies and Design Faculty, Inonu Caddesi no 65, Gumussuyu 34437- Beyoglu-Istanbul-Turkey;

${ }^{4}$ Textile Engineering Department, Faculty of Technology, Goztepe Istanbul, 34722, Turkey;

${ }^{*}$ Corresponding Author: dawood@neduet.edu.pk

\section{Abstract:}

A test rig is described, for the measurement of temperature and resistance parameters of a Temperature Sensing Fabric (TSF) for calibration purpose. The equipment incorporated a temperature-controlled hotplate, two copper plates, eight thermocouples, a temperature data-logger and a four-wire high-resolution resistance measuring multimeter. The copper plates were positioned above and below the TSF and in physical contact with its surfaces, so that a uniform thermal environment might be provided. The temperature of TSF was estimated by the measurement of temperature profiles of the two copper plates. Temperature-resistance graphs were created for all the tests, which were carried out over the range of 20 to $50^{\circ} \mathrm{C}$, and they showed that the temperature and resistance values were not only repeatable but also reproducible, with only minor variations. The comparative analysis between the temperature-resistance test data and the temperature-resistance reference profile showed that the error in estimation of temperature of the sensing element was less than $\pm 0.2^{\circ} \mathrm{C}$. It was also found that the rig not only provided a stable and homogenous thermal environment but also offered the capability of accurately measuring the temperature and resistance parameters. The Temperature Sensing Fabric is suitable for integration into garments for continuous measurement of human body temperature in clinical and non-clinical settings.

\section{Keywords:}

temperature sensor, resistance temperature detector, temperature sensing fabric, test-rig for temperature-resistance calibration, textile sensors, human body temperature

\section{Introduction}

In recent years, intensive research has been carried out to extend the scope of ambulatory monitoring from exclusively clinical to non-clinical environments [1-3]. The mobile monitoring of various vital signs of the human body requires sensors to be integrated into a next-to-skin smart garment. In standard medical settings, the vital signs of the human body include body temperature, respiratory activity, electrocardiography (ECG) and blood pressure [4]. Previous research has predominantly focused on the development of textile based respiration, movement and electrocardiography sensors [5-11]. For the temperature measurement of human body, researchers have mainly relied on commercially manufactured external temperature sensors, such as temperature-measuring semiconductor devices and also thermistors [12-15]. In order to address such shortcomings, a detailed study has been carried out for the development of a textile based temperature sensor (also termed a Temperature Sensing Fabric), which might maintain intimate contact with the surface of skin and facilitate long-term physiological monitoring of human body [16-20].

The details of design and fabrication of Temperature Sensing Fabric (TSF) are readily available [17]; the sensing fabric was fabricated on a computerized industrial flat-bed knitting machine by embedding a fine metallic wire as a sensing element in the middle of the double layer knitted structure, as shown in the conceptual TSF illustrated in Figure 1. The copper, nickel and tungsten sensing elements, in the form of bare and insulated wires of various diameters, were used in the fabrication of the TSF to identify the most suitable design. The working principle of the TSF is based on the fundamental tendency of metal wire to vary its electrical resistance when exposed to a change of temperature. The TSF samples were developed by embedding 3.8 meters of sensing wire into an $8 \times 8 \mathrm{~cm}^{2}$ temperature monitoring area. In order to avoid an electrical contact between the adjacent inlaid wires, additional spacer courses were also introduced, along with the knitted courses. As the metal wire was embedded in the middle of a double layer knitted structure, it was hardly noticeable and did not significantly affect the aesthetics or the comfort of the fabric. The compact double layer structure of the TSF also provided protection from abrasion. This work details the design, development and performance of the test rig system, which was employed to calibrate the TSF samples over a temperature range from $20^{\circ} \mathrm{C}$ to $50^{\circ} \mathrm{C}$.

Calibration of the TSF is prerequisite before deploying it for the measurement of human body skin temperature. No published material has been found in which a standard procedure or equipment suitable for the calibration of temperature sensing fabric has been reported. Usually, a Resistance Temperature Detector (RTD) is calibrated in a primary or secondary temperature monitoring laboratory using either fixed point or comparison methods [21]. The calibration technique adopted 
depends upon the accuracy requirement and the temperature range. Such calibration methods are usually performed for wide temperature ranges when non-linearity becomes important. Fixed point calibration is performed for primary standard thermometers by the use of fixed point cells in order to attain the lowest possible measurement uncertainty; it is an expensive and time consuming process. Comparison calibrations are performed by using a standard reference thermometer in conjunction with a dry or liquid temperature-controlled bath. This is a less expensive process and can be performed either in a specialized laboratory or at the deployment site. In both types of calibration, sophisticated resistance bridges are used to measure the electrical resistance; however, for both the comparison and the fixed point calibration techniques, the sensor to be calibrated is immersed either in a liquid thermal environment or in a dry thermal environment comprising standard sized metal blocks. Considering the construction and the constituent materials of Temperature Sensing Fabric, a liquid thermal environment is not appropriate, and similarly, the shape and compliance of a TSF renders it incompatible with the dimensions of the standard sized metal blocks used for calibration in dry thermal environments.

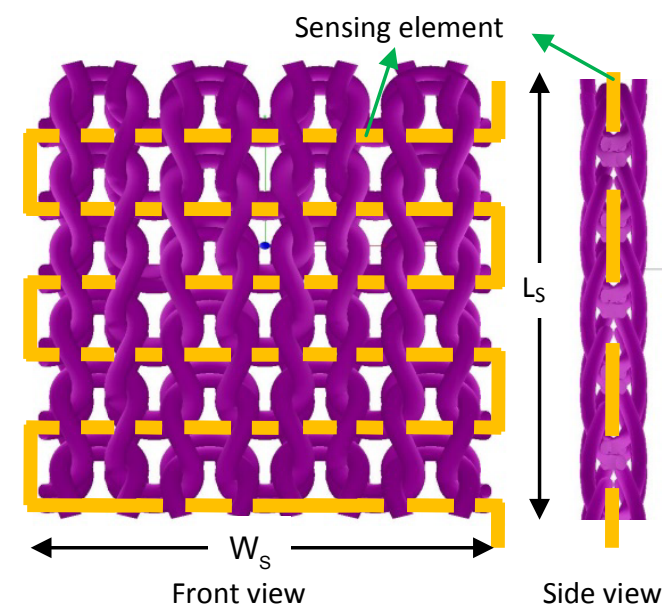

Figure 1. A conceptual illustration of the knitted sensor showing the embedded sensing wire

According to standard temperature laboratories [22, 23], the dedicated fixed point cells or metal blocks could be adapted to calibrate theTemperature Sensing Fabric up to $300^{\circ} \mathrm{C}$ with a calibration accuracy of $\pm 0.1^{\circ} \mathrm{C}$ by considering any nonlinearities. However, it is important to note that the relevant temperature range of the application environment extends from only 20 to $50^{\circ} \mathrm{C}$. Moreover, errors associated with the construction of the TSF and its application environment (movement artefacts, the influence of environmental parameters, etc.) may be significant. It is therefore inappropriate to initiate the design of a sophisticated temperature-measuring system, as high precision is unlikely to be warranted. Therefore, the development of the test rig along with the calibration procedure at an appropriate level of accuracy and precision was a core objective of this research. The TSF samples may be calibrated by investigating the effect of temperature on their resistance. In order to quantify this effect, an ohm-meter and reliable temperature sensors plus a heating system to artificially create a thermal environment at the required temperature, were considered to be the primary requirements of the system.
The proposed testing methodology is based on contact temperature measurement across the TSF coupled with four wire resistance measurement. The equipment employs a constant temperature hotplate in order to maintain the TSF at a series of stable temperatures, and includes two copper plates between which the TSF is sandwiched so that the sensor temperature might be derived without direct measurement. Each copper plate was instrumented with four K-type thermocouples and a PicoTech TC-08 data logger was used for recording the temperature. The temperature of the sample was approximated by averaging the temperatures of the upper and lower copper plates. An Agilent 34401A, 6.5-digit multimeter and 4-wire resistance measurement system were used to measure the changes in electrical resistance. A purpose-built graphical user interface was created in the LabVIEW environment to record and display the temperature and resistance signals side by side.

\section{Materials \& Methods}

\subsection{General Requirement of Test Rig}

The primary purpose of the test rig was to record the electrical resistance of the sensing element and the corresponding temperature values of the Temperature Sensing Fabric in order to develop an empirical relationship between resistance and temperature for calibration purposes. Following were the general requirements of the test rig: creation of a uniform thermal environment for the TSF within a temperature range of $20^{\circ} \mathrm{C}$ to $50^{\circ} \mathrm{C}$; accurate temperature measurement of the TSF by temperature sensor via the contact measurement method; and the measurement of minute changes in resistance of the TSF, as a consequence of change in temperature by attachment of multimeter leads to the sensing element.

Since the observations involved the measurement of precise levels of resistance and temperature, the contact quality of the test rig settings was therefore important and needed to be uniform in all the testing procedures. The primary concern was how to present a uniform thermal environment to the TSF at specified temperature settings. Further technical difficulties to be considered included the attachment of temperature sensors to the sensing element (of the TSF), the compensation of the additional resistance introduced by the multimeter leads when they were connected into the measuring circuit and the nullification of errors in the perceived resistance of the TSF which might arise due to the effect of test rig temperature fluctuations on the multimeter leads. There were also procedural issues to address, in particular, the minimization of handling the equipment and the sensing fabric during the testing procedure.

\section{2 Preliminary approaches to measure Temperature and Resistance}

Accurate temperature measurement of the TSF (either its surface or the embedded sensing wire) may not be as straightforward as it might appear. The TSF consists of three interacting components, that is, air, textile and metal wire. Each component has different thermal properties and unless the TSF 
is in a steady state, accurate temperature measurement of the sensing element may not be possible.

In the earlier stages of this study, the temperature measurement of the TSF was performed within various thermal environments between 20 and $50^{\circ} \mathrm{C}$, by using a variety of techniques, that is, contact based and non-contact based temperature measuring methods. Initially, a handheld infrared temperature measuring instrument was employed to measure the temperature of a bare wire sensing element. However, large variations in temperature were observed because of the small Field of View offered by the wire. Similarly, the measurement of surface temperature of the TSF was also found to be less stable and more inaccurate because of the surface characteristics of the TSF. Due to surface roughness, emissivity values may not be constant and can influence the infrared spectrum. Additionally, it was also not possible to use this instrument for continuous measurement.

Temperatures were also measured by contact method, that is, by attaching the temperature sensors (thermocouple and thermistor) at the surface of TSF or directly onto the sensing element. Under uniform thermal conditions of $50^{\circ} \mathrm{C}$, the surface temperature of the TSF was measured with variations of $\pm 3^{\circ} \mathrm{C}$. Similarly, direct temperature measurement of the sensing element was also not consistent and this resulted in large variations. The main problem in both cases was the quality of contact between the temperature sensor and the TSF surface (or sensing element). The quality of contact could be improved by permanent fastening of the temperature sensor onto the sensing element (e. g. , soldering) or at the surface of the TSF (e. g. , gluing). However, this invasive approach could not be endorsed as the quality of thermal contact would not be the same for different samples and would be a source of variation.

Initial modelling and experimental results indicated that a $1^{\circ} \mathrm{C}$ change in temperature caused a resistance change, in the sensing element of the order of milliohms $(\mathrm{m} \Omega)$, and was particularly small when the nominal resistance of the TSF was low. Measuring such a low resistance accurately is not a straightforward task. In preliminary testing, the resistance was measured using a standard hand-held multimeter with a crocodile clip, however, significant variations were observed due to the lead resistance, contact resistance and limitations in the precision of the hand-heldmultimeter.

\subsection{Development of Test Rig Methodology}

It was understood from the preliminary testing that a tailor-made non-invasive test rig coupled with a consistent procedure, with minimized manual handling, had to be developed. The test rig should not only provide a uniform thermal environment at the desired temperature but should also measure the TSF temperature and its corresponding resistance accurately.

Therefore, a purpose-built test rig was developed around a number of commercially available components.

- A TC-08 Data logger by PicoTech was used to measure and record temperature readings via thermocouples, as shown in Figure 2. Up to eight thermocouples can be connected into a single data-logging unit. It offers a temperature measurement accuracy of $\pm 0.2 \%$ of the reading plus $\pm 0.5^{\circ} \mathrm{C}$ and interfaces to a PC using USB cable [24].

- The Agilent 34401A is an industry standard benchtop digital multimeter, as depicted in Figure 2, which provides a combination of high resolution, accuracy and speed. Its measurement capability includes 6.5 digits of resolution with $0.01 \%$ ohm measurement accuracy. For resistance measurement, the multimeter offers a maximum resolution of $100 \mu \Omega$ on its $100 \Omega$ range and it interfaces with the PC via GPIB or RS232 connectors [25].
A



E

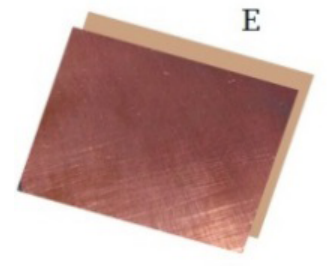

D

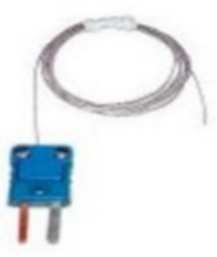

B

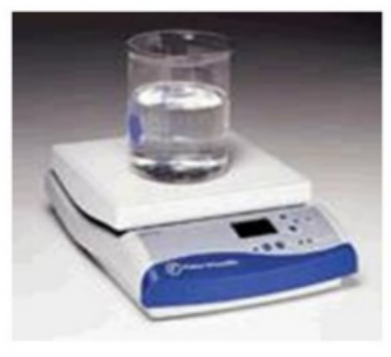

C

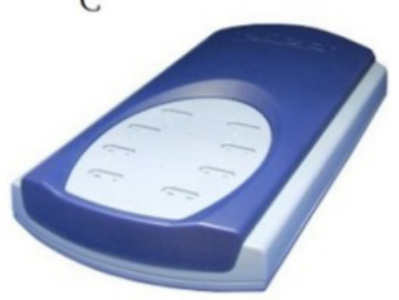

Figure 2. Test rig components: A) Agilent 34401 multimeter; B) Fischer hotplate; C) PicoTech TC-08 data logger; D) K type thermocouple; E) Copper plates. 
-A Fischer Scientific Hotplate was used as a heating source for the test rig as it provides contact heat to the samples. Its top plate $(7 \times 7$ in. $)$ is made of reflective white ceramic, providing a maximum of $540^{\circ} \mathrm{C}$ for its temperature setting. An LED display shows the actual and set point temperatures which can be adjusted with nominal increments of $1^{\circ} \mathrm{C}$ [26].

\section{Thermal Environment Provision \& Temperature Measurement}

In order to provide a uniform thermal environment for the TSF and to measure its resistance at each corresponding temperature step, a new approach was worked out by understanding and adapting the methodology of the standard parallel hotplate method for measurement of the thermal resistance of textiles [27]. In this method, two highly conductive metal plates would not only provide a uniform thermal environment to a sample sandwiched between them, as shown in Figure 3, but would also provide a temperature sensing platform. The lower metal plate would be placed on the surface of a standard laboratory hotplate. Heat energy would transfer via conduction from the hotplate to the environment by passing through the lower metal plate, the sample under test (TSF) and the upper metal plate, respectively. By measuring the temperatures of the upper and lower metal plates, the temperature of the sample could be approximated.

\section{Copper Plate Development}

Copper was chosen for the metal plates because of its high thermal conductivity and ease of availability. Copper plates were developed not only to provide a consistent thermal environment but also to measure the temperature across the TSF as shown in Figure 3. In order to provide a uniform thermal environment to the samples, it is important that the temperature distribution between the metal plates is uniform axially and radially at steady temperature settings between 20 and $50^{\circ} \mathrm{C}$. Therefore, along with the choice of material, the thickness of the copper plates was an important consideration. In order to improve the uniformity of temperature within each copper plate, it was desirable to select the thinnest possible metal sheets. However, it was also wished to fasten the thermocouples in the middle of the copper plates for sensing purposes. Therefore, the thickness of the metal plates was decided by considering the thickness of thermocouples. A copper plate of $4 \mathrm{~mm}$ thickness would not only be able to securely embed the $2 \mathrm{~mm}$ thermocouple probe but because of the plate's low Biot number [28], it also exhibits excellent temperature homogeneity.

Each copper plate was fitted with four k-type thermocouples as shown in Figure 3. The thermocouples were fixed with thermally conductive adhesive, into shaped grooves, so that

A)

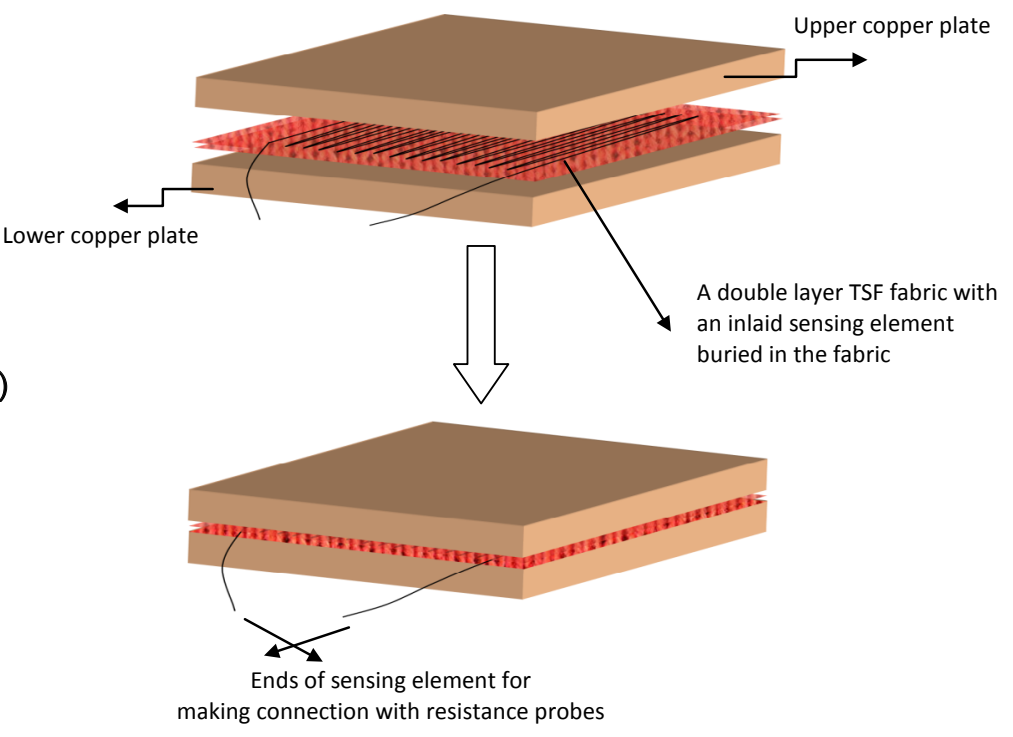

B)

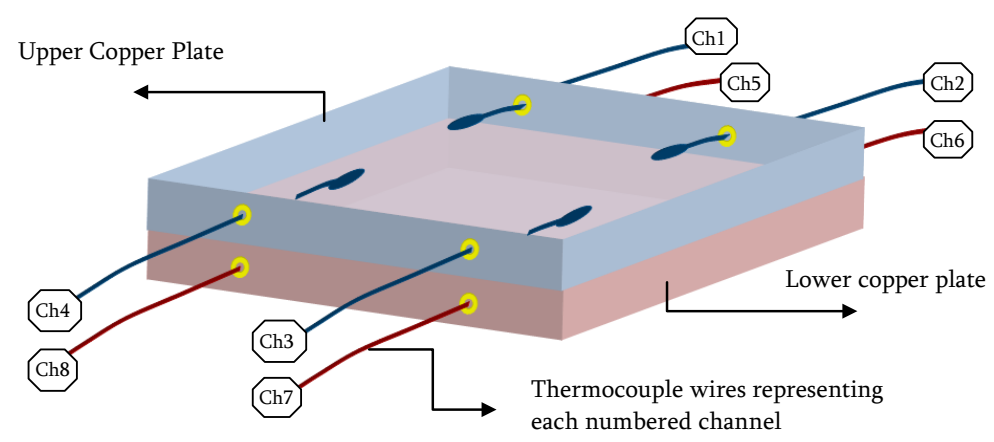

Figure 3. A) Placement of TSF between the upper and lower copper plates, B) Position of thermocouples in the upper and lower copper plates 
the thermocouple probe would have an optimal contact with the copper plates. This connection was further protected by fixing a thin copper strip on the same side of the copper plate, where the thermocouples were fastened; the overall thickness of the copper plates was $4 \mathrm{~mm}$. The temperature data logger could acquire signals from the eight thermocouples simultaneously, so by averaging the eight thermocouples, a close approximation could be determined of the temperature of the sensing element embedded in the middle of the TSF.

\section{Resistance Measurement}

As a result of preliminary testing of TSF resistance, a number of concerns were identified as being the main contributors of error in the resistance measurement and needed to be rectified:

- the inherent resistance of the multimeter leads;

- the quality of contact between the multimeter probes and the ends of the sensing element;

- the effect of temperature on the resistance of the multimeter leads.

These issues were resolved by taking the following actions:

- introducing Four-Wire Resistance Measurement;

- developing a special connector for Four-Wire Resistance Measurement;

- using a high precision benchtop multimeter instead of a handheld instrument.

Two-Wire Resistance measurement involves passing a current through an electrical component and measuring the voltage drop across the target device. This entails the use of two connecting leads, one being coupled to each end of the target component [29]. The method is illustrated by the topleft circuit in Figure 4 and gives acceptable accuracy under normal circumstances. Moreover, handling two probes is straightforward and taking readings is simple and relatively

A)
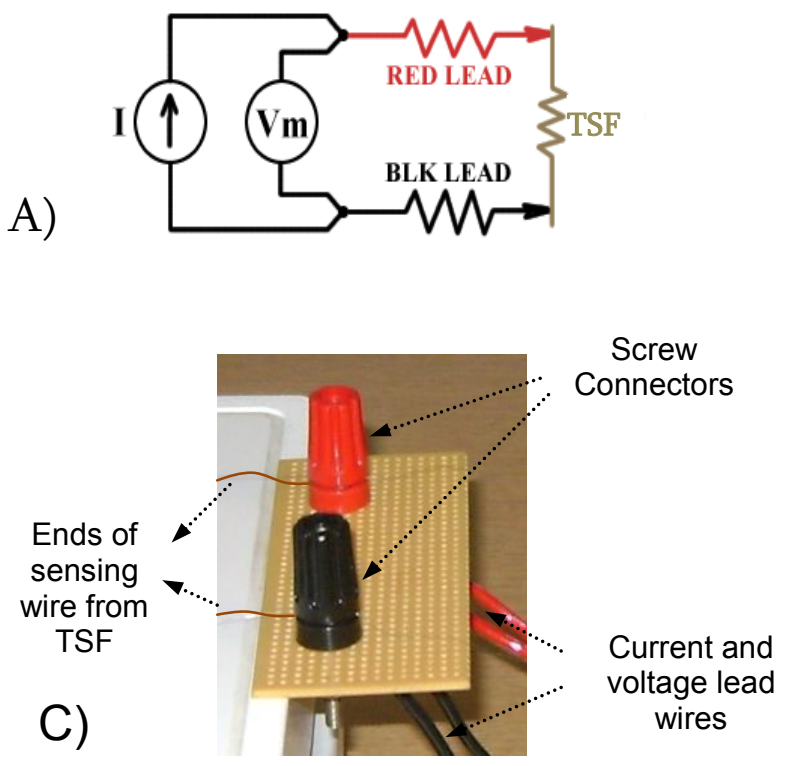

Figure 4. Resistance Measurement system

A) Circuit diagram of two wire resistance measurement

B) Circuit diagram of four wire resistance measurement

C) Customized connector to measure resistance by four-wire method

D) Multimeter with current and voltage lead wires quick. Another long-established approach for accomplishing this task is Four-Wire Resistance Measurement (4WRM), which provides a much more accurate way to measure small resistances. The offsetting effects of lead resistances and contact resistances are automatically reduced using this method. In 4WRM, two leads are used to pass current, whilst a second pair of leads is used to measure the voltage drop as shown in the top-right circuit in Figure 4 [28].

Thus, the requirement was to develop a 4WRM system to take reliable resistance measurements. The 4WRM connections were created by fixing substantial screw type connectors onto an insulating strip and then soldering the current and voltage lead wires to the underside of the connector as shown in Figure 4. A firm contact between the sensing element of the TSF and the four-wire connector can be made from the upper side using a screwed terminal. This approach minimizes the resistance contribution of the connecting lead, and thus, resolves the issues of resistance offset and resistance variation with temperature change in the connecting leads. During temperature-resistance testing, it is important to exercise care to ensure that the sensing element does not make contact with the surface of either of the conducting copper plates while sandwiching the TSF between them, or while making connections to the 4WRM connector. Any contact between the sensing element and the copper plates during the testing procedure would lead to error in the resistance measurement.

In order to record the TSF temperature and its corresponding resistance, a customized graphical user interface was also developed in the LabVIEW environment. A complete setup of the test rig system and the LabVIEW interface is pictured in Figure 5.

The test rig apparatus is a simplified version of a standard parallel hotplate method for the measurement of thermal
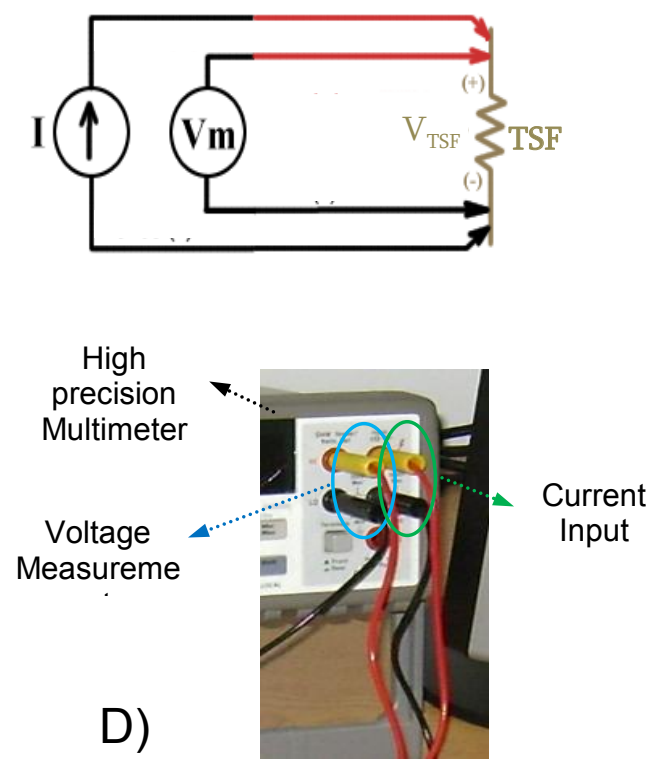


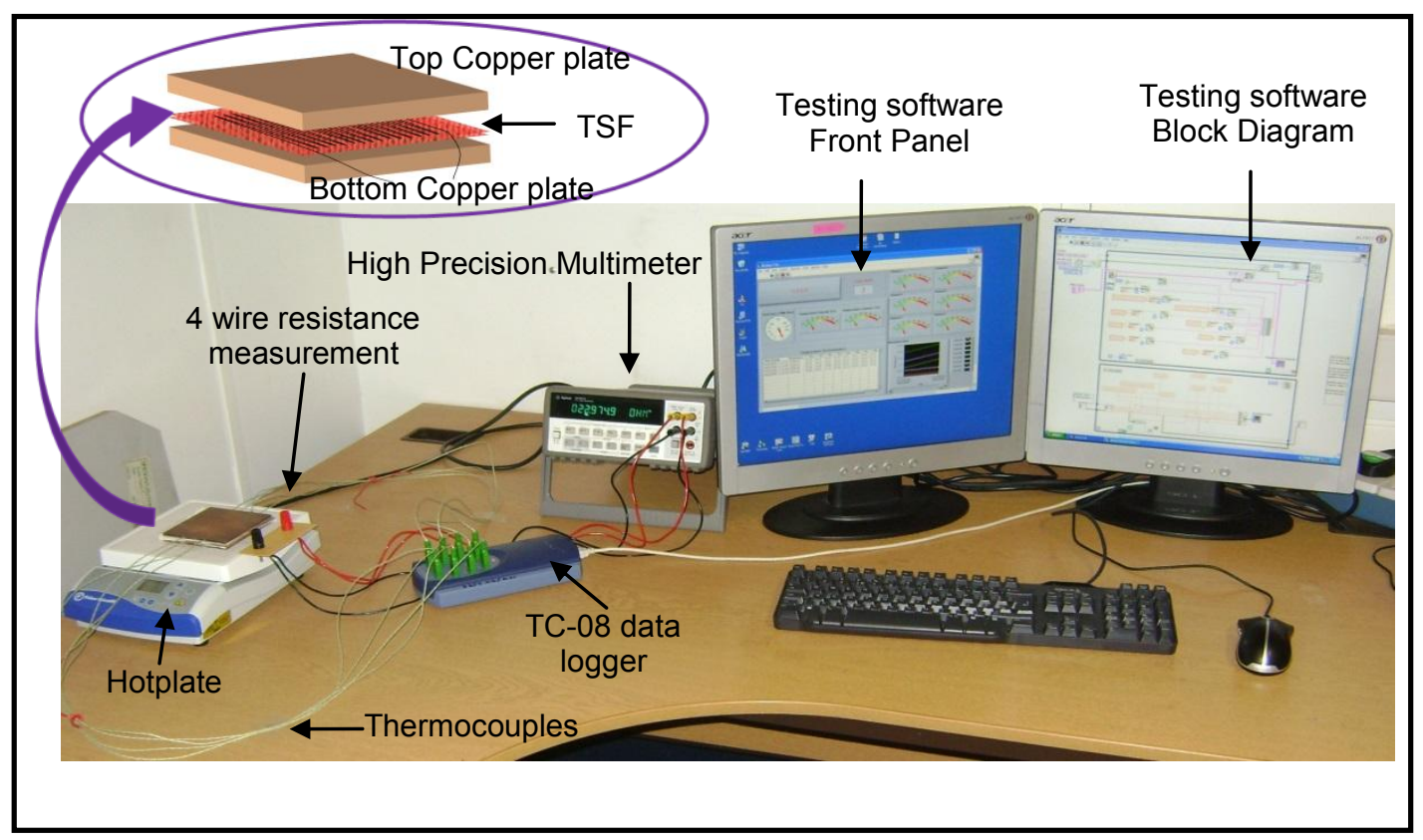

Figure 5. Photograph of the test rig while in operation.

resistance of textiles [27]. In the standard parallel hotplate method, metal plates and samples are insulated along the edges in order to minimize radial heat transfer. This is important for the measurement of thermal conductivity (or thermal resistance) of material under steady state conditions. However, this experimental test apparatus is not insulated along the sides, as such a refinement was not considered necessary in view of the level of accuracy expected from this type of sensor under the conditions in which a wearable sensor is expected to be used. In the earlier stages of the research, an attempt was made to insulate the rig components along the edges, however, because of the handling procedure for the test rig, a permanent solution of fixed insulation was not suitable, as it had to be regularly removed and replaced, suffering handling damage each time. During the changing of the sample in the test rig, the top copper plate has to be removed from its position. At this moment, the thermocouple wires attached to the copper plates and the sensing element of the TSF interfered with any insulating material fitted along the sides of the plates. This meant that the side insulation had to be reset each time a sample was changed; the effectiveness of the insulation was therefore a little different for each test, thereby adding slight variations to the result. Therefore, to improve the consistency of testing, and to minimize handling problems with the TSF samples, side insulation was not incorporated into the design.

\section{Results and Discussion}

Before starting the actual temperature-resistance testing, it was necessary to perform a number of preliminary tests in order to establish the limitations of the testing regime and to estimate the inaccuracies by understanding the test rig behavior. It was important to ascertain the extent to which it might be possible to make a close approximation to the actual temperature of the sensing element. It was equally important to discover whether the temperature throughout the whole sample would be uniform. The factors which might be capable of disturbing the thermal environment of the test rig would also have to be found. It was also important to find whether the test rig could record actual $T-R$ values at certain temperature points.

TSF samples may be calibrated by investigating the effect of temperature on their resistance and the TemperatureResistance relationship of pure metals is relatively linear within the temperature range between 0 and $100^{\circ} \mathrm{C}[17,30]$. This means that suitable calibration equations may be created by specifying the temperature-resistance data values at two fixed points. One of the fixed pointsmay be considered to be the standard laboratory temperature of $20^{\circ} \mathrm{C}$, while the second point could be above the normal temperature of the human body, that is, $37^{\circ} \mathrm{C}$. Therefore, tests were performed within a stable environment of $45^{\circ} \mathrm{C}$. The resistance values drawn at $20^{\circ} \mathrm{C}$ and $45^{\circ} \mathrm{C}$ would help devise the calibration equation. Creating a stable $45^{\circ} \mathrm{C}$ environment for the TSF and then measuring the temperature and resistance values would be more challenging in comparison to the data acquisition at room temperature $\left(20^{\circ} \mathrm{C}\right)$. Therefore, forthcoming experiments will discuss the measurement results and estimation of errors at the highest value of the selected range, that is, at $45^{\circ} \mathrm{C}$.

After various assessment attempts, an operational hotplate temperature of $55^{\circ} \mathrm{C}$ was chosen, as this resulted in a stable TSF temperature close to $45^{\circ} \mathrm{C}$. The measured surface temperatures are usually less than the actual surface temperature primarily because of environmental influences and the contact resistance between the temperature sensor and the surface to be measured. In order to estimate this error in the test rig setting, both the upper and lower copper plates were placed on the hotplate without any fabric sample. At an indicated hotplate temperature of $55^{\circ} \mathrm{C}$, the average temperature of the top and bottom copper plates was recorded by the eight thermocouples as being $49^{\circ} \mathrm{C}$ and $49.5^{\circ} \mathrm{C}$ respectively. The $0.5^{\circ} \mathrm{C}$ drop in temperature between the copper plates was 
primarily due tothe contact thermal resistance. The $5.5^{\circ} \mathrm{C}$ drop in temperature between the hotplate and lower copper plate could be consequential upon various factors. It was believed that the indicated temperature of the hotplate was actually the temperature of the internal heating elements located just underneath the hotplate surface. Due to the thermal contact resistance between the hotplate's internal heating elements and the surface, the displayed temperature would always be little more than the actual surface temperature of the hotplate. This means that a $5.5^{\circ} \mathrm{C}$ drop in temperature between the hotplate and the lower copper plate was partially because of the contact thermal resistance between the hotplate surface and the lower copper plate, and also the contact thermal resistance between the hotplate's internal heating elements and its own surface.

A few tests were carried out to ascertain the uncertainty in measuring the temperature of the upper and lower copper plates, and to assess the stability of the thermal environment between the plates at the indicated temperature of the hotplate. In this test, a sample of TSF was placed in between the two copper plates and then the hotplate was turned on with an indicated temperature of $55^{\circ} \mathrm{C}$. The temperature readings of both copper plates were noted for about half an hour once the test rig components achieved equilibrium.

The detailed results of this experiment are presented in Figure 6 and Table 1. Figure 6 shows the temperature profiles of all eight thermocouples, the average for the upper and lower copper plates and the TSF temperature. The average temperature of each thermocouple channel, the standard deviation for each channel, the average temperature of each copper plate and the standard deviation among the channels of each copper plate were also calculated and presented in Table 1.

The standard deviation within each temperature channel was found to be extremely low, which means that the thermocouples and the data acquisition system are high in precision. The standard deviation among all the channels of their respective copper plate was calculated to be less than $0.2^{\circ} \mathrm{C}$. All the thermocouples belonging to each respective copper plate showed the same behavior and their average relative differences were noted to be less than $0.5^{\circ} \mathrm{C}$. This means that due to the high thermal conductance of the copper plates, the temperature distribution within each plate was uniform and there was no significant temperature gradient within the plates. It is implicit from these figures that both copper plates provided a stable thermal environment for the TSF at the $55^{\circ} \mathrm{C}$ set temperature of the hotplate.

To confirm the reproducibility of the results, four more tests were carried out on different days at the standard laboratory temperature of $20^{\circ} \mathrm{C}$. The TSF temperature profiles of all five tests along with their corresponding resistance profiles are shown in Figure 7. The temperature values of all five tests were found to be in the range from $45.30^{\circ} \mathrm{C}$ to $45.55^{\circ} \mathrm{C}$ with a standard deviation of 0.06 . The resistance values of all five tests were measured to be in the range from $51.45 \Omega$ to $51.55 \Omega$, with a standard deviation of 0.013 . It can be seen from Figure 7 that temperature and resistance values are not only repeatable but reproducible as well, with only very small variations. This means

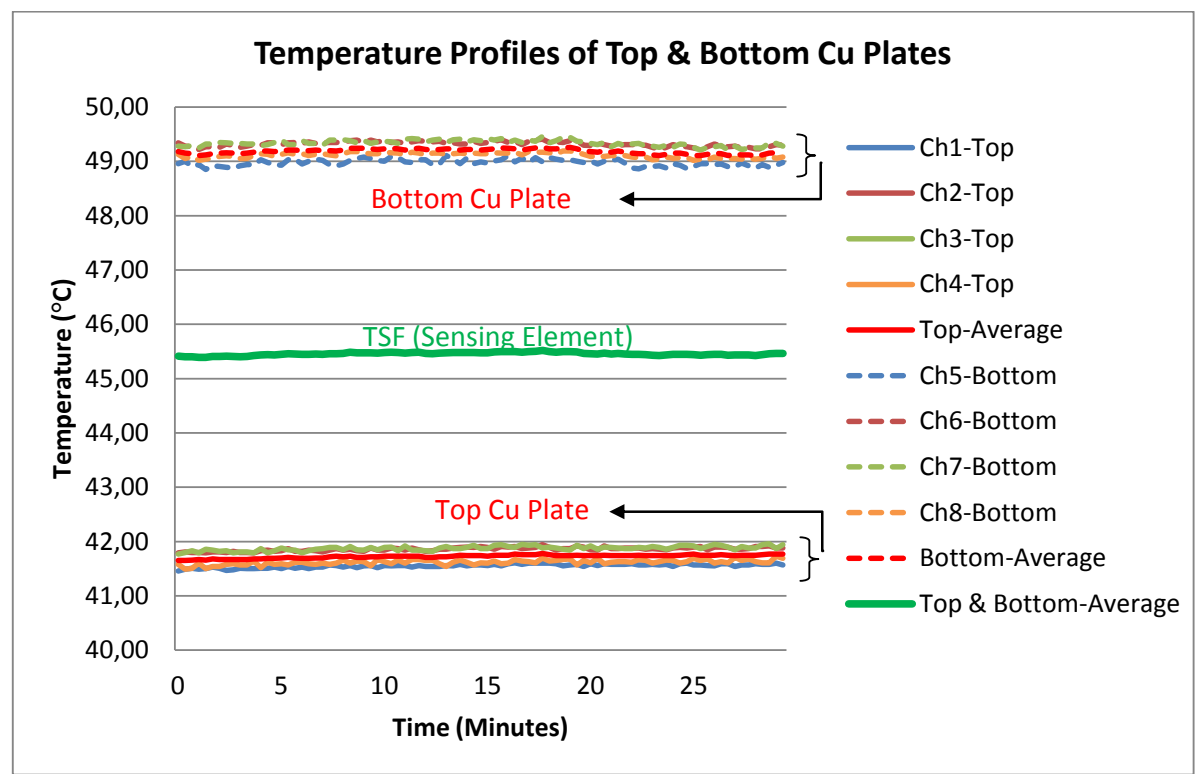

Figure 6. Temperature profiles of the upper and lower copper plates at a nominal $55^{\circ} \mathrm{C}$ hotplate temperature.

Table 1. Averages and standard deviations of the temperature profiles of the upper and lower copper plates.

\begin{tabular}{|c|c|c|c|c|c|c|c|c|}
\hline Thermocouple Channel number & Ch1-Top & Ch2-Top & Ch3-Top & Ch4-Top & Ch5-Bottom & \begin{tabular}{|l|} 
Ch6-Bottom \\
\end{tabular} & Ch7-Bottom & Ch8-Bottom \\
\hline Average of each channel & 41.55 & 41.86 & 41.87 & 41.62 & 48.97 & 49.32 & 49.34 & 49.11 \\
\hline \multirow[t]{2}{*}{ Std. Dev. within each channel } & 0.03 & 0.04 & 0.04 & 0.05 & 0.06 & 0.05 & 0.06 & 0.05 \\
\hline & \multicolumn{3}{|c|}{ Average Top } & 41.73 & \multicolumn{3}{|c|}{ Average Bottom } & 49.19 \\
\hline \multicolumn{2}{|c|}{ Standard deviation within all channels of Top Cu plate } & 0.16 & \multicolumn{5}{|c|}{ Standard deviation within all channels of Bottom Cu plate } & 0.17 \\
\hline
\end{tabular}


A

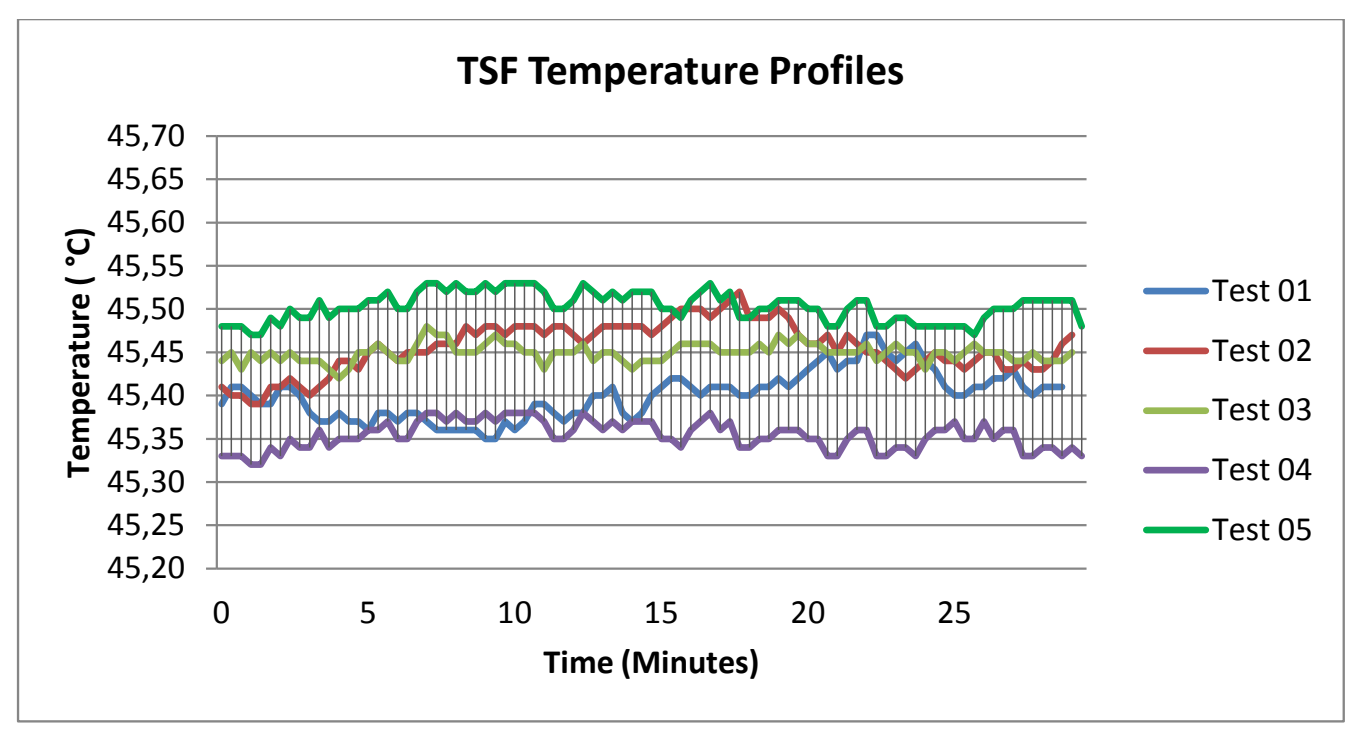

B

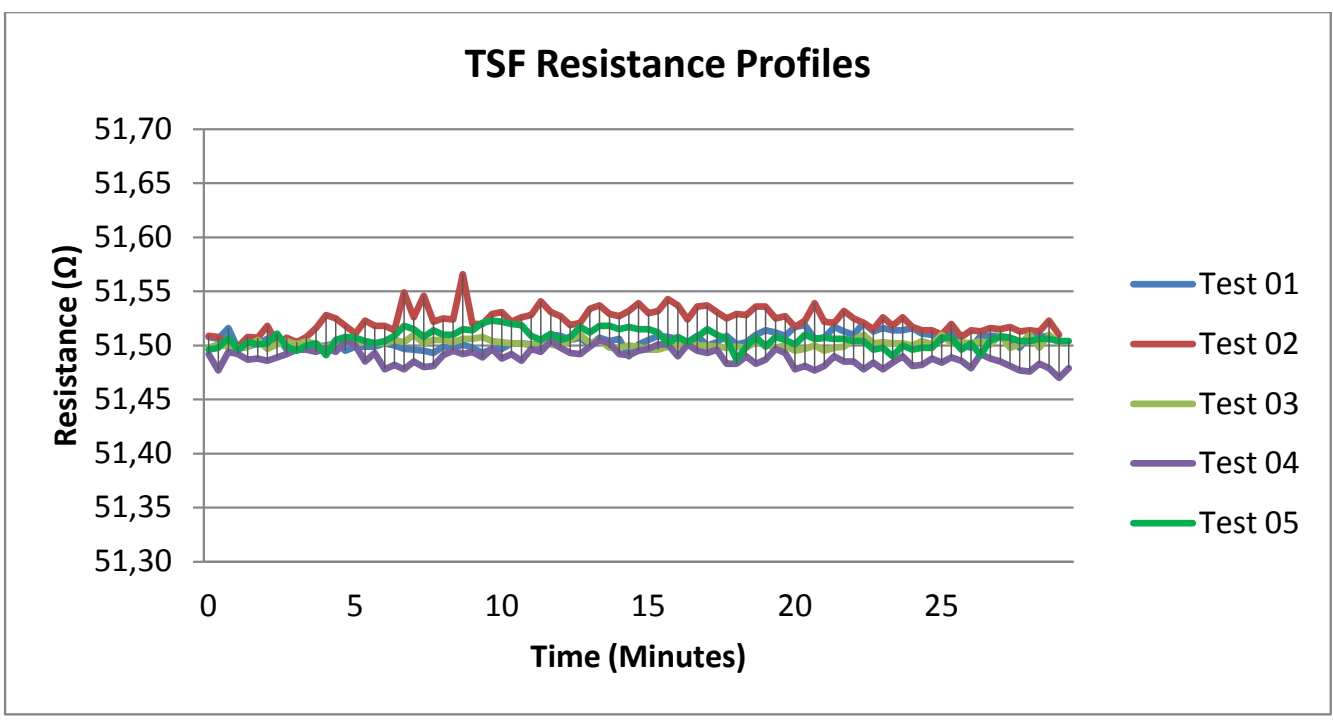

Figure 7. TSF profiles at a nominal set temperature of the hotplate of $55^{\circ} \mathrm{C}$. A) TSF Temperature profiles B) TSF Resistance profiles

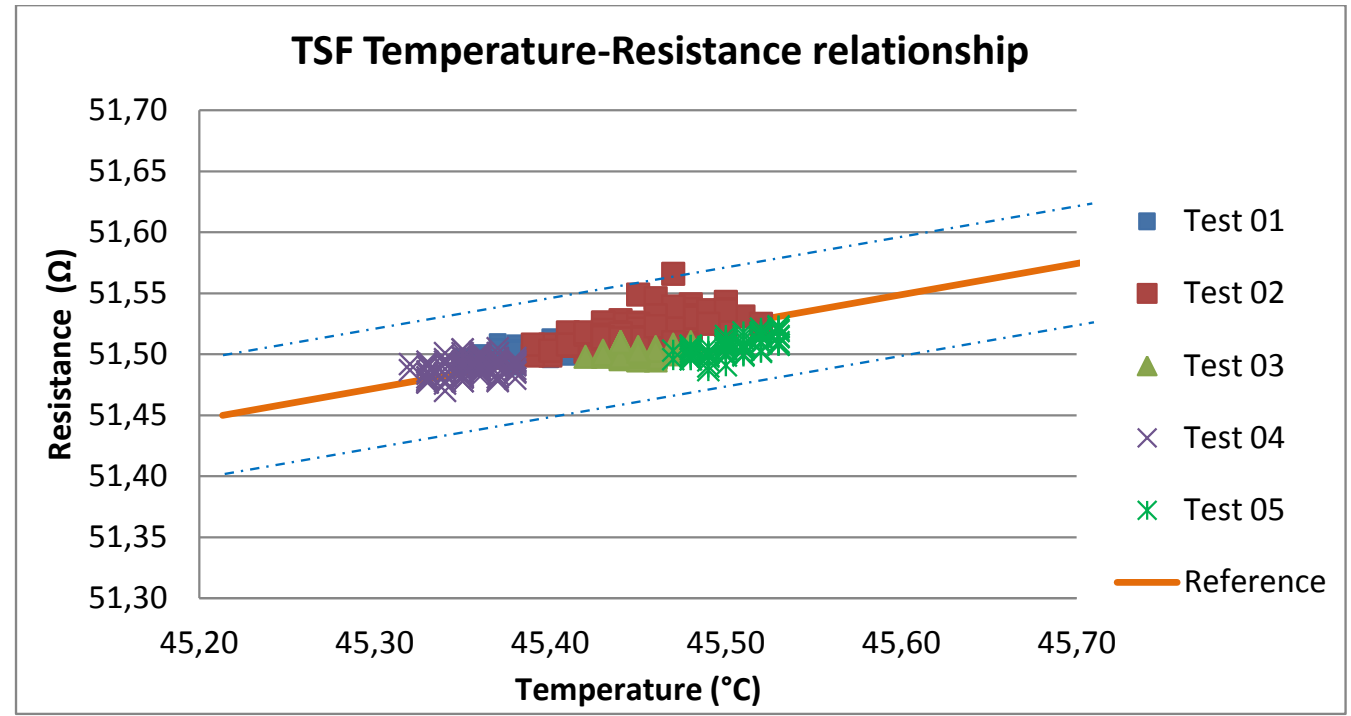

Figure 8. TSF temperature-resistance profiles at a nominal $55^{\circ} \mathrm{C}$ set temperature of the hotplate. 
that the test rig not only provided a stable and homogenous thermal environment but also has the capability to precisely measure the temperature and resistance values.

It was important to determine how accurately the temperature of the sensing element (embedded in the TSF) can be estimated within the temperature range between 20 and $50^{\circ} \mathrm{C}$ for the test rig. The temperature measurement methodology, that is, the estimation of the sensing element temperature by taking an average of the upper and lower temperature of the copper plates may seem straight forward to understand. However, it was necessary to validate it by scientific investigation and to estimate the errors in calculating the sensing element temperature. The methodology was based on the assumptions that: the sensing element is embedded exactly in the middle of the TSF; the temperature varies linearly across the thickness of the TSF; the thermal contact resistance and drop in temperature at the interfaces of TSF surfaces to their respective copper plates are the same. Deviation from these assumptions may contribute errors in the estimation of temperature of the sensing element. In terms of TSF structure, not only the positioning of the sensing element is important but also the homogeneity of the textile layers across the sensing element.

In order to validate the methodology, the TemperatureResistance data values of all five tests were compared with the reference Temperature-Resistance profile, as shown in Figure 8. The specifications of the TSF and its reference Temperature-Resistance profile are stated in Table 2. It can be seen from Figure 8, that the Temperature-Resistance data are randomly distributed across the reference profile. The maximum deviation from the reference line is not more than $\pm 50 \mathrm{~m} \Omega$, which corresponds to an error of $\pm 0.20^{\circ} \mathrm{C}$. At the room temperature $\left(20^{\circ} \mathrm{C}\right)$, the resistance deviation from the reference line was measured to be less than $\pm 20 \mathrm{~m} \Omega$, which corresponds to an error of $\pm 0.08^{\circ} \mathrm{C}$. So, it is safe to say that the overall error at specified temperature range would be not more than $\pm 0.20^{\circ} \mathrm{C}$. With reference to the above discussion, it can be inferred that the temperature of the wire can be closely approximated (with maximum error of $\pm 0.20^{\circ} \mathrm{C}$ ) by taking the average temperature of the upper and lower copper plates within the range from $20^{\circ} \mathrm{C}$ to $50^{\circ} \mathrm{C}$.

\section{Conclusions}

This research reports in detail on the design, development and behavioral analysis of a test rig designed to measure the temperature and resistance parameters of a Temperature Sensing Fabric (TSF) over the range from $20^{\circ} \mathrm{C}$ to $50^{\circ} \mathrm{C}$. The testing methodology was based on contact temperature measurement across the TSF and utilized four wire measurement of resistance. The results indicate that although there were significant heat losses from the system, the high level of conductance of the $4 \mathrm{~mm}$ copper plates located within a temperature controlled laboratory maintained a very even temperature across the plates. This, coupled with their relatively high thermal capacity, served to counter the thermal cycling of the temperature control system built into the hotplate, so that the TSF fabric could be maintained at a series of stable and repeatable test temperatures. Hence, the temperature of the fabric surface might be calculated without resorting to direct surface measurement of the textile sensor.

The thermocouples built into the copper plates allied with the 6.5-digit multimeter and the 4-wire resistance measurement system proved to be able to accurately measure and record the minute changes in temperature and electrical resistance of the TSF. It was found that the temperature distribution within each copper plate, at a nominal $55^{\circ} \mathrm{C}$ preset hotplate temperature, was uniform without significant temperature gradient. The standard deviation within each temperature channel was $0.06^{\circ} \mathrm{C}$ and within the resistance channel was $0.013 \Omega$; this was extremely small and indicated that the sensors and the data acquisition system operated at high precision. The temperatureresistance graphs of all tests showed that the temperature and

Table 2. The specifications of the TSF and its reference Temperature-Resistance profile.

\begin{tabular}{|l|c|}
\hline Parameter & Value \\
\hline Sample Description & $\begin{array}{c}\text { An } 8 \mathrm{~cm} \times 8 \mathrm{~cm} \text { TSF sample embedded } \\
\text { with Nickel } 270 \text { sensing element of } 90 \\
\text { micron diameter. }\end{array}$ \\
\hline Sensing Element X-Area & $6.36 \mathrm{E}-09\left(\mathrm{~m}^{2}\right)$ \\
\hline Sensing Element Alpha (Temperature Coefficient of Resistivity) $\left[0^{\circ} \mathrm{C}-100^{\circ} \mathrm{C}\right]$ & $0.0064\left(1 /{ }^{\circ} \mathrm{C}\right)^{\circ}$ \\
\hline Rho (Resistivity) @ $20^{\circ} \mathrm{C}$ & $7.45 \mathrm{E}-08 \Omega \mathrm{m}^{\diamond}$ \\
\hline Length of Sensing Element embedded in the TSF & $3.85(\mathrm{~m})$ \\
\hline Nominal Resistance @ $0{ }^{\circ} \mathrm{C}$ & $39.90(\Omega)$ \\
\hline Sensitivity & $256\left(\mathrm{~m} \Omega /{ }^{\circ} \mathrm{C}\right)$ \\
\hline Resistance/Meter @ $20^{\circ} \mathrm{C}$ & $11.7(\Omega / \mathrm{m})$ \\
\hline Resistance Ratio $\left[20-50^{\circ} \mathrm{C}\right]$ & 1.17 \\
\hline Reference Equation & $\mathrm{R}=0.256^{*} \mathrm{~T}+39.896$ \\
\hline
\end{tabular}


resistance values were not only repeatable but reproducible as well, with only very small variations. The comparative analysis between the temperature-resistance test data and the temperature-resistance reference profile showed that the error in the estimation of the temperature of the temperature sensing fabric was not more than $\pm 0.2^{\circ} \mathrm{C}$. It may therefore be inferred that the rig provided a stable and homogenous thermal environment; it also provided the capability to precisely measure the temperature and resistance parameters. In addition, it may also be concluded that over a temperature range extending from ambient temperature of $20^{\circ} \mathrm{C}$ up to $50^{\circ} \mathrm{C}$, the temperature of the sensing wire may be closely approximated by averaging the temperatures of the upper and lower copper plates. The temperature sensing fabric may therefore be integrated into garments for accurate continuous measurement of human body temperature in mobile, non-clinical settings, for example, sports, military, general healthcare and firefighting situations and this will facilitate studies related to biorhythms and assessment of thermal strain in extreme environments.

\section{ACKNOWLEDGMENTS}

The authors would like to acknowledge the funding provided by the following organizations to carry out this study at the School of Materials, University of Manchester, Manchester, UK:

- NED University of Engineering \& Technology Pakistan, through the Higher Education Commission of Pakistan; and - Ministry of National Education of Turkey

\section{References}

[1] Baig, M. , H. Gholamhosseini, and M. Connolly, A comprehensive survey of wearable and wireless ECG monitoring systems for older adults. Medical \& Biological Engineering \& Computing, 2013. 51(5): p. 485-495.

[2] Young Hwan, K. , et al. A robust wearable health monitoring system based on WSN. in Consumer Communications and Networking Conference (CCNC), 2013 IEEE. 2013.

[3] Stoppa, M. and A. Chiolerio, Wearable Electronics and Smart Textiles: A Critical Review. Sensors, 2014. 14(7): $p$. 11957-11992.

[4] Funnell, R. , G. Koutoukidis, and K. Lawrence, Chapter 21 Vital Signs, in Tabbner's nursing care: theory and practice. 2009, Churchill Livingstone: Australia. p. 251-274.

[5] Atalay, O. and W. Kennon, Knitted Strain Sensors: Impact of Design Parameters on Sensing Properties. Sensors, 2014. 14(3): p. 4712-4730.

[6] Atalay, O. , W. Kennon, and M. Husain, Textile-Based Weft Knitted Strain Sensors: Effect of Fabric Parameters on Sensor Properties. Sensors, 2013. 13(8): p. 11114-11127.

[7] Paul, G. , et al. , Novel active electrodes for ECG monitoring on woven textiles fabricated by screen and stencil printing. Sensors and Actuators A: Physical, 2015. 221(0): p. 60-66.

[8] Catrysse, M. , et al. , Towards the integration of textile sensors in a wireless monitoring suit. Sensors and Actuators A: Physical, 2004. 114(2-3): p. 302-311.

[9] Chang, W. Y., T. H. Fang, and Y. C. Lin, Characterization and fabrication of wireless flexible physiological monitor sensor. Sensors and Actuators A: Physical, 2008. 143(2): p. 196-203.
[10] Mazzoldi, A. , et al. , Smart Textiles for Wearable Motion Capture Systems. AUTEX Research Journal, 2002. 2(4).

[11] Atalay, O. , W. R. Kennon, and E. Demirok, Weft-Knitted Strain Sensor for Monitoring Respiratory Rate and Its Electro-Mechanical Modeling. Sensors Journal, IEEE, 2015. 15(1): p. 110-122.

[12] Curone, D. , et al. Smart Garments for Emergency Operators: Results of Laboratory and Field Tests. in 30th Annual International IEEE Engineering in Medicine and Biology Society (EMBS) Conference. 2008. Vancouver, British Columbia, Canada.

[13] Derchak, P. A. , K. L. Ostertag, and M. A. Coyle, LifeShirt ( System as a monitor of heat stress and dehydration. 2004: VivoMetrics, Inc. Ventura, CA

[14] Noury, N. , et al. VTAMN - A Smart Clothe for Ambulatory Remote Monitoring of Physiological Parameters and Activity. in 26th Annual International IEEE Engineering in Medicine and Biology Society (EMBS) Conference. 2004. San Francisco, California.

[15] Pandian, P. S. , et al. , Smart Vest: Wearable multiparameter remote physiological monitoring system. Medical Engineering \& Physics, 2008. 30: p. 466-477.

[16] Husain, M. D. and W. R. Kennon, Preliminary Investigations into the Development of Textile Based Temperature Sensor for Healthcare Applications. Fibers, 2013. 1(1): p. 2-10.

[17] Husain, M. D. , W. R. Kennon, and T. Dias, Design and Fabrication of Temperature Sensing Fabric. Journal of Industrial Textiles, 2014. 44(3): p. 398-417.

[18] Husain, M. D. , O. Atalay, and W. R. Kennon, Effect of Strain and Humidity on the Performance of Temperature Sensing Fabric. International Journal of Textile Science, 2013. 2(4): p. 105-112.

[19] Husain, M.D., et al., Measuring Human Body Temperature through Temperature Sensing Fabric. AATCC Journal of Research, 2016. 3(4): p. 1-12.

[20] Husain, M.D., et al., Uncertainty Analysis of the Temperature-Resistance Relationship of Temperature Sensing Fabric. Fibers, 2016. 4(4): p. 29.

[21]Ayres, D. and A. Blundell, Calibrating Thermometers, Isothermal Technology Limited.

[22] NPL. Contact Thermometry Section, National Physical Laboratory, UK. 08-07-2014. http://www.npl.co.uk/ temperature-humidity/.

[23] ISOTECH. Isothermal Technology Limited, UK. 08-072014. http://www.isotech.co.uk.

[24] PicoTech. USB TC-08 Thermocouple Data Logger. 08-072014. http://www. picotech.com/thermocouple.html.

[25] Agilent Technologies. Agilent 34401A Multimeter - Product Overview. 08-05-2015. http://cp.literature.agilent.com/ litweb/pdf/5968-0162EN.pdf.

[26] Fischer Scientific. Digital *Isotemp* Hotplates. 08-07-2014. http://www.fishersci.com.

[27]BS 4745, Determination of the thermal resistance of textiles - Two-plate method: fixed pressure procedure, two-plate method: fixed opening procedure, and singleplate method. 2005.

[28] Cengel, Y. A. , Chapter 4 - Transient Heat Conduction, in Heat Transfer: A Practical approach. 2003, McGraw-Hill. p. 209-264.

[29] Michalski, L. , et al. , Chapter 4 - Resistance Thermometers, in Temperature Measurement. 2002, John Wiley \& Sons, Ltd. p. 85-102.

[30] Childs, P. R. N. , Chapter 6 - Resistance Temperature Detectors, in Practical Temperature Measurement. 2001, Butterworth-Heinemann. p. 145-193. 